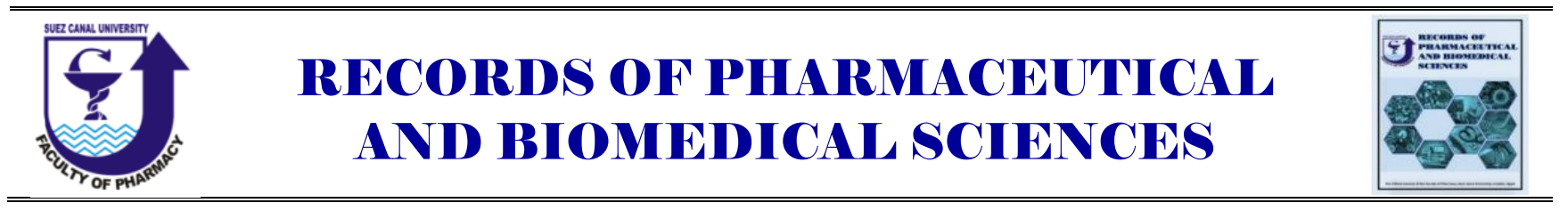

\title{
Multidrug resistant Klebsiella pneumoniae isolated from respiratory tract infection
}

\author{
Randa Khattab $^{a^{*}}$, Ghada Shaker ${ }^{b}$, M'Omen Askoura $^{b}$, Hisham Abbas $^{b}$ \\ ${ }^{a}$ Police authority hospital, ministry of health, Egypt, \\ ${ }^{b}$ Department of Microbiology and Immunology, Faculty of Pharmacy, Zagazig University, Egypt
}

Received on: 17.08. 2019

Revised on: 09. 09. 2019

Accepted on: 13. 09. 2019

Correspondence Author:

Tel:+201001099937

E-mail address:

Dr.RandaHesham@yahoo.com

\begin{abstract}
An increasing antimicrobial resistance among Klebsiella pneumoniae ( $K$. pneumoniae) has been rapidly growing. The cross-sectional, analytical and descriptive study was conducted to investigate the multidrug resistance (MDR) of $K$. pneumoniae isolated from respiratory tract infection. One hundred and fifty-one $K$. pneumoniae isolates were recovered from 330 sputum specimens $(45.75 \%)$. The isolates showed high levels of resistance to the most of antibiotics and intermediate resistance prevalence against imipenem and meropenem. Random and misuse of antibiotics lead to the emergence of superbugs. Prospective studies must focus on new strategies for facing of the tsunami of drug resistance.
\end{abstract}

Keywords: Klebsiella pneumoniae, Multidrug resistance, Respiratory tract infection, Egypt

\section{Introduction}

K. pneumoniae is a Gram-negative bacterium, belongs to family Enterobacteriaceae. $K$. pneumoniae can be stated as the major cause of different types of lower respiratory infections followed by septicemia. The majority of the infections were diagnosed in hospitalized patients, it indicates that $K$. pneumoniae is a potent nosocomial pathogen in our hospitals(Kramer et al., 2006).

Differences in clinical features of $K$. pneumoniae infections are related with nature and number of expressed virulence factors. $K$. pneumoniae has the potential to cause mainly respiratory, urinary, blood and wound infections and recently, liver abscess syndrome was widely reported in Asian countries, it is associated with high virulence strains of capsular serotype K1 or K2 with hypermucoviscosity phenotype resulting mainly from K1-specific gene and a plasmid-borne gene (Ravichitra et al., 2014)

Treatment of infections caused by $K$. pneumoniae is hindered by emergence of bacterial resistance to antimicrobial agents. $K$. pneumoniae is capable of employing a multitude of mechanisms by which to confer resistance to most available antibiotics, efflux pumps play a major role. Bacterial efflux pumps are membrane proteins that decrease intracellular concentration of antimicrobial agents through pumping them outside the cells. Importantly, bacterial efflux pumps are nonspecific in action and therefore could mediate bacterial resistance to structurally unrelated antimicrobial agents (Nahar and Rashid, 2017). Increasing the efflux also plays a role, especially with hydrophobic compounds that presumably enter the cell via diffusion (Silver et al., 1977). 
At the same speed where these antimicrobials are entering the cell, efflux mechanisms are pumping them out again, before they reach their target (Nahar and Rashid, 2017).

Multidrug resistance proteins or Multidrug efflux pumps exist in almost all bacterial species. Multidrug efflux pumps are divided into five classes according to their different compositions, energy sources, substrates, and number of transmembrane domains: (i) the Major Facilitator Superfamily; (ii) the Resistance-Nodulation-Division family; (iii) the ATP-Binding Cassette superfamily; (iv) the Small Multidrug Resistance family; and (v) the Multidrug And Toxic compound Extrusion family (Algowaihi et al., 2016). In addition to antibiotics, multidrug efflux pumps are also found to expel a broad range of environmental and physiological toxic compounds, such as dyes, detergents, bile acids, hormones, organic acids, etc. Thus, they are also referred to as xenobiotic pumps or transporters (Algowaihi et al., 2016).

The widespread emergence of MDR bacterial pathogens is an important public health concern worldwide (WHO, 2014). K. pneumoniae is one of these MDR organisms identified as an urgent threat to human health its infections are particularly a problem among neonates, elderly and immunocompromised individuals within healthcare setting (Miller et al., 2007).

The aim of the present study was to determine the prevalence of $K$. pneumoniae from patients with respiratory tract infections and to investigate MDR and efflux pump activity in K. pneumoniae isolated from respiratory tract infection.

\section{Material and Methods}

\subsection{Study design and specimen collection}

A total of three hundred and thirty clinical sputum and tracheal swab specimens were collected over the period from September 2017 to October 2018. From the collected specimens 151 samples selected to study antimicrobial resistance of $K$. pneumoniae. These specimens were collected from patients admitted to Zagazig and Tanta University Hospitals. The specimens were obtained from patients in department of chest section, internal medicine, neural section, neurological care, anesthesia care, emergency anesthesia care and oncology. All specimens were collected aseptically and transported to the microbiology laboratory, where they were immediately processed according to standard microbiological procedure (Winn, 2006).

\subsection{Isolation and identification of isolates}

The samples were cultured by the streaking on blood agar, nutrient agar and MacConkey agar and then incubated at a $37^{\circ} \mathrm{C}$ for 24 hours. The bacterial colonies were purified and identified according to colonies morphology. API-20E system (bioMerieux) was used for identification of $K$. pneumoniae isolates.

\subsection{Antimicrobial susceptibility testing}

Antimicrobial susceptibilities of $K$. pneumoniae isolates to different antimicrobial agents were determined using Kirby-Bauer disk diffusion method on Mueller-Hinton agar following the Clinical and Laboratory Standards Institute (Andrews, 2001) guidelines. A number of 14 antimicrobial discs, representing different classes of antimicrobial agents, were included in this study. The discs were the product of Oxoid, UK: imipenem $(10 \mu \mathrm{g})$, meropenem $(10 \mu \mathrm{g})$, amoxicillin $(10 \mu \mathrm{g})$, amoxicillin on / clavulanate $(20 / 10 \mu \mathrm{g})$, ampicillin/sulbactam $(20 \mu \mathrm{g})$, piperacillin / tazobactam $(100 / 10 \mu \mathrm{g})$, cefepime $\quad(30$ $\mu \mathrm{g})$,cefotaxime $(30 \mu \mathrm{g})$, cefaclor $(30 \mu \mathrm{g})$, amikacin $(30 \mu \mathrm{g})$,gentamicin $(10 \mu \mathrm{g})$, azithromycin $(15 \mu \mathrm{g})$, ciprofloxacin $(5 \mu \mathrm{g})$, tetracycline $(10 \mu \mathrm{g})$,

A colony from each $K$. pneumoniae isolate was grown overnight in Mueller Hinton broth (Oxoid, UK) at $37^{\circ} \mathrm{C}$. Bacterial cultures were adjusted to be equivalent to 0.5 MacFarland standard $(1.5 \mathrm{x}$ $108 \mathrm{CFU} / \mathrm{ml}$ ) using Micro-Scan Turbidity Meter and plated on Mueller Hinton agar (Oxoid, UK) by the streaking method using a sterile swab. Antibiotic discs were applied using fine-end forceps and plates incubated at inverted position at $37^{\circ} \mathrm{C}$ for $16-18 \mathrm{hrs}$. Inhibition zone were measured and interpreted as sensitive (S), intermediate (I) or resistant (R) according to CLSI criteria (Clinical and Laboratory Standards, 2015).

Isolates that show resistance to at least three different classes of antimicrobial agents were considered as MDR.

\subsection{Statistical analysis}

Wilcoxon rank-sum test was used for identifying significant differences between study groups of antibiotics. $P$ value $<0.05$ in all tests this showed that the results are statistically significant. 


\section{Results}

\subsection{Study cohort}

One hundred and fifty-one $K$. pneumoniae isolates were recovered from 330 specimens. Patients' gender was 98 males and 63 females 53, of the 151 specimens that were positive for Klebsiella.

\subsection{Antimicrobial susceptibility testing}

K. pneumoniae isolates showed high resistance to cefaclor, amoxicillin, ampicillin -sulbactam, cefotaxime, azithromycin, ciprofloxacin, amoxicillin - clavulanic acid, Gentamicin, cefepime, amikacin, cefoperazone - Sulbactam and Piperacillin - Tazobactam, while intermediate resistance prevalence against imipenem and Meropenem (Tables 1,2).

\section{Discussion}

K. pneumoniae is a Gram-negative, non-motile, encapsulated, lactose fermenting, facultative anaerobic, rod shaped bacterium found in the normal flora of the mouth, skin and intestines. In the recent years, $K$. pneumoniae has become important pathogen in nosocomial infections (Pitout et al., 2015).

It was observed that of the positive Klebsiella isolates, $66 \%$ were from males while $34 \%$ from females indicating that males show greater vulnerability for these infections (Aly and Balkhy, 2012). K. pneumoniae is an important cause of MDR infections worldwide. Epidemiological studies have highlighted the emergence of MDR and $K$. pneumoniae strains isolated from hospitalacquired infections (AlZubiery et al., 2018).

Table 1. Susceptibility patterns of MDR isolates to different antimicrobial agents

\begin{tabular}{|c|c|c|c|c|c|c|}
\hline \multirow{3}{*}{ Antimicrobials agents } & \multicolumn{6}{|c|}{ Susceptibility interpretation of $K$. pneumoniae isolates $(\mathrm{n}=151)$} \\
\hline & \multicolumn{2}{|c|}{ (R) } & \multicolumn{2}{|c|}{ (I) } & \multicolumn{2}{|c|}{ (S) } \\
\hline & No & $\%$ & No & $\%$ & No & $\%$ \\
\hline Imipenem & 48 & 31.79 & 20 & 13.25 & 83 & 54.97 \\
\hline Meropenem & 72 & 47.68 & 15 & 9.93 & 64 & 42.38 \\
\hline Amoxicillin & 142 & 94.04 & 0 & 0 & 9 & 5.96 \\
\hline Amoxicillin, Clavulanic acid & 122 & 80.79 & 13 & 8.61 & 16 & 10.6 \\
\hline Ampicillin - Sulbactam & 142 & 94.04 & 4 & 2.65 & 5 & 3.31 \\
\hline Piperacillin - Tazobactam & 100 & 66.23 & 18 & 11.92 & 33 & 21.85 \\
\hline Cefepime & 116 & 76.82 & 7 & 4.64 & 28 & 18.54 \\
\hline Cefotaxime & 134 & 88.74 & 5 & 3.31 & 12 & 7.95 \\
\hline Cefaclor & 146 & 96.69 & 2 & 1.32 & 3 & 1.99 \\
\hline Cefoperazone - Sulbactam & 114 & 75.5 & 6 & 3.97 & 31 & 20.53 \\
\hline Amikacin & 108 & 71.52 & 10 & 6.62 & 33 & 21.85 \\
\hline Gentamicin & 117 & 77.48 & 9 & 5.96 & 25 & 16.56 \\
\hline Azithromycin & 133 & 88.08 & 11 & 7.28 & 7 & 4.64 \\
\hline Ciprofloxacin & 126 & 83.44 & 6 & 3.97 & 18 & 11.92 \\
\hline
\end{tabular}

(No) number of isolates; (R) resistant; (I) intermediate; (S) sensitive 
Table 2. Resistance profile of $K$. pneumoniae isolates

\begin{tabular}{lcc}
\hline \multicolumn{1}{c}{$\begin{array}{c}\text { Resistance profile of } \text { K. pneumoniae isolates to various } \\
\text { antimicrobial agents. }\end{array}$} & $\begin{array}{c}\text { Number of } \\
\text { resistant isolates }\end{array}$ & \% \\
\hline Sensitive to all antimicrobial agents & 1 & 0.66 \\
Resistance to one antimicrobial agent & 2 & 1.32 \\
Resistance to two antimicrobial agents & 2 & 1.32 \\
Resistance to three antimicrobial agents & 5 & 3.31 \\
Resistance to four antimicrobial agents & 7 & 4.64 \\
Resistance to five antimicrobial agents & 10 & 6.62 \\
Resistance to six antimicrobial agents & 16 & 10.60 \\
Resistance to seven antimicrobial agents & 22 & 14.57 \\
Resistance to eight antimicrobial agents & 25 & 16.56 \\
Resistance to nine antimicrobial agents & 61 & 40.40 \\
Total & 151 & 100 \\
\hline
\end{tabular}

The induced efflux pump activity is responsible for the increased antibiotic resistance in $K$. pneumoniae. The most significant $(\mathrm{P}<0.05)$ finding in this study was that $K$. pneumoniae treated with sequential antibiotics, showed reduced susceptibility towards all antibiotics in study except imipenem and Meropenem, leading to the multiple antibiotic resistance.

Therefore, antibiotic therapies should take into account the history of pre-exposed antibiotics to prevent the development of antibiotic resistance.

The increasing resistance to antibiotics by respiratory pathogens has complicated the use of empirical treatment with traditional agents and a definitive bacteriological diagnosis and susceptibility testing would, therefore, be required for effective management of Lower respiratory tract infection (Chakraborty et al., 2016). MDRs are often associated with extended spectrum betalactamase producing bacteria, that is, resistance to other classes of drugs like aminoglycosides and quinolones (Ravichitra et al., 2014).

There was an observed high resistant antibiotic profile to ampicillin (80.6\%) and amoxicillin (74.2\%). All K. pneumoniae isolates are naturally resistant to amoxicillin and ampicillin, due to a constitutively expressed chromosomal class-A ßlactamase (Giske et al., 2012)

\section{Conclusion}

This study highlights the influence of using sequential antibiotic treatment on the development of antibiotic resistance in $K$. pneumoniae. These findings necessitate continuous monitoring of antimicrobial resistance to guide the empirical treatment of $K$. pneumoniae diseases. High antibiotic resistance of $K$. pneumoniae towards commonly used antibiotics are the major reasons for misuse of antibiotics, antibiotics are not prescribed based on sensitivity testing, the wide use of broad-spectrum antibiotics, prolonged infections, increased hospitalization, increased the cost of therapy and enhanced morbidity and mortality rates.

\section{References}

Algowaihi R, Ashgar S, Sirag B, Shalam S, Nassir $A$ and Ahmed AJGA (2016). Draft genome sequence of a multidrug-resistant Klebsiella pneumoniae strain isolated from King Abdullah Medical City, Makkah, Saudi Arabia. 4, e0037516.

Aly M and Balkhy H (2012). The prevalence of antimicrobial resistance in clinical isolates from Gulf Corporation Council countries. Antimicrob Resist Infect Control. ; 1 (1): 26. 
Alzubiery T, Alharazi T, Alsumairy H, Al-Zubeiry A, Yusr A and Al-Shami Huda ALJSJBR (2018). Current Antibiotic Sensitivity Pattern of Clinically Isolated Klebsiella pneumoniae. 3, 23-32.

Andrews JMJJOaC (2001). Determination of minimum inhibitory concentrations. 48, 5-16.

Chakraborty S, Mohsina K, Sarker PK, Alam M, Karim M and Sayem SJPB (2016). Prevalence, antibiotic susceptibility profiles and ESBL production in Klebsiella pneumoniae and Klebsiella oxytoca among hospitalized patients. 118, 53-58.

Clinical and Laboratory Standards I (2015). M100S25 Performance Standards for Antimicrobial Susceptibility Testing; Twenty-Fifth Informational Supplement. CLSI 35, 1-240.

Giske CG, Fröding I, Hasan CM, et al. (2012). Diverse sequence types of Klebsiella pneumoniae contribute to the dissemination of blaNDM-1 in India, Sweden, and the United Kingdom. 56, 27352738.

Kramer A, Schwebke I and Kampf GJBID (2006). How long do nosocomial pathogens persist on inanimate surfaces? A systematic review. 6, 130 .

Miller LG, Quan C, Shay A, et al. (2007). A prospective investigation of outcomes after hospital discharge for endemic, community-acquired methicillin-resistant and-susceptible Staphylococcus aureus skin infection. Clinical Infectious Diseases 44, 483-492.
Nahar N and Rashid RBJDUJOPS (2017) Phylogenetic Analysis of Antibiotic Resistance Genes and Virulence Genes of Klebsiella species in silico. 16, 119-127.

Pitout JDD, Nordmann P and Poirel L (2015). Carbapenemase-producing Klebsiella pneumoniae, a key pathogen set for global nosocomial dominance. Antimicrobial agents and chemotherapy 59, 5873-5884.

Ravichitra KN, Prakash PH, Subbarayudu S and Rao US (2014). Isolation and antibiotic sensitivity of Klebsiella pneumoniae from pus, sputum and urine samples. Int. J. Curr. Microbiol. App. Sci 3, 115-119.

Silver L, Chandler M, De La Tour EB and Caro L (1977). Origin and direction of replication of the drug resistance plasmid R100. 1 and of a resistance transfer factor derivative in synchronized cultures. Journal of bacteriology 131, 929-942.

Winn WC (2006). Koneman's color atlas and textbook of diagnostic microbiology.(Lippincott williams \& wilkins).

World Health Organization (2014):

(http://who.int/world-healthday/2014/policybriefs/en/index.html 